\title{
Parallelism Effects and Verb Activation: The Sustained Reactivation Hypothesis
}

\author{
Sarah M. Callahan · Lewis P. Shapiro • Tracy Love
}

Published online: 23 September 2009

(C) The Author(s) 2009. This article is published with open access at Springerlink.com

\begin{abstract}
This study investigated the processes underlying parallelism by evaluating the activation of a parallel element (i.e., a verb) throughout and-coordinated sentences. Four points were tested: (1) approximately $1,600 \mathrm{~ms}$ after the verb in the first conjunct (PP1), (2) immediately following the conjunction (PP2), (3) approximately $1,100 \mathrm{~ms}$ after the conjunction (PP3), (4) at the end of the second conjunct (PP4). The results revealed no activation at PP1, suggesting activation related to the initial presentation had decayed by this point; however, activation was observed at PP2, PP3, and PP4, suggesting the conjunction elicits reactivation that is sustained throughout the second conjunct. These findings support a specific hypothesis about parallelism, the sustained reactivation hypothesis. This hypothesis claims that, in conjoined structures, a cue that is associated with parallelism elicits the reactivation of material from the first conjunct and that this activation is sustained until integration with the second conjunct can be completed.
\end{abstract}

Keywords Parallelism $\cdot$ Verb processing $\cdot$ Activation $\cdot$ Reactivation $\cdot$ Sentence processing

\section{Introduction}

This paper describes a study involving parallelism, a particular type of restricted relation between two conjoined elements. We first define what we mean by parallelism and then briefly review the psycholinguistic literature that suggests that, given two conjoined phrases or clauses, processing of the second is facilitated when it is parallel to the first on any of a variety of relevant dimensions. We then describe two experiments that chart the time-course

S. M. Callahan $(\bowtie) \cdot$ T. Love

University of California, San Diego, 9500 Gilman Drive,

Mail Code 0109, La Jolla, CA 92093-0109, USA

e-mail: smcallahan@ucsd.edu

L. P. Shapiro · T. Love

San Diego State University, San Diego, CA, USA 
of activation of a parallel element across two conjoined clauses, and interpret our data to suggest that parallelism results in a reactivation of material from the first clause.

With respect to language processing, the term, parallelism, refers to a similarity at one or more levels of linguistic structure. Parallelism can occur at various levels of structure ranging from phonology to syntax to semantics to discourse. Coordinated elements (e.g., elements joined by the conjunction, and) are the prototypical environment for parallelism and it is well-known that language comprehenders (i.e., listeners and readers) prefer coordinate structures to be parallel. The investigation of the role of parallelism in processing is in a relatively early stage. In 1984, Frazier and colleagues published the first systematic investigation of the effects of parallelism on processing (Frazier et al. 1984), revealing that the second conjunct of two coordinated sentences was read faster when it was parallel to the first conjunct in terms of syntactic structure, as in (1), than when it was nonparallel, as in (2).

(1) The tall gangster hit John and the short thug hit Sam.

(2) The tall gangster hit John and Sam was hit by the short thug.

(Frazier et al. 1984)

This facilitation in processing (i.e., faster reading times for the second of two parallel as compared to non-parallel elements) has been dubbed the "parallelism effect". Since the original report by Frazier and colleagues, researchers have investigated parallelism effects with the goal of building a successful theory of parallelism. Parallelism in syntactic structure (i.e., syntactic parallelism) has received the most study-to-date, but effects of parallelism in phonological structure (Carlson 2001), discourse structure (Kehler 2002), and semantic structure (Frazier et al. 1984; Knoeferle and Crocker 2009) have also been observed. In the following section, previous research is summarized in terms of its import for the fundamental aspects of such a theory: namely, the scope of parallelism, the types of representations it operates upon, and the types of processes that are involved.

\section{Previous Research}

In the first report of parallelism effects on the processing of coordinate structures (Frazier et al. 1984), Frazier and colleagues reported a self-paced reading study that compared reading times for second conjuncts that were parallel or non-parallel to the first conjunct on one of several dimensions of syntactic or semantic structure (e.g., voice, attachment site, thematic roles, animacy). Strikingly, faster reading times were observed for the second conjunct when the conjuncts were parallel on any of the dimensions tested. A subsequent eye-tracking study (Frazier et al. 2000) investigated reading times for the second of two conjuncts and demonstrated faster total (but not first-pass) reading times ${ }^{1}$ for that region when it was parallel to the first conjunct in terms of internal structure (i.e., whether or not a noun phrase was modified by an adjective). In that study, similar parallelism effects were also observed for comparisons of coordinate structures where the parallel and non-parallel versions were both rated as fully acceptable.

More recently, Knoeferle and colleagues extended findings of parallelism effects to German (Apel et al. 2007; Knoeferle and Crocker 2009). In a series of eye-tracking experiments (Knoeferle and Crocker 2009) the constituent order of noun phrases in coordinated sentences (i.e., the order of the subject and object) was manipulated. In sentences such as (3)

\footnotetext{
1 In eye-tracking studies, first-pass reading time refers to the sum of all fixations in a region from the first fixation in that region until the first fixation in any other region and reflects initial reading. Total reading time refers to the sum of all the fixations in a region and reflects both initial reading and re-reading.
} 
below (with regions for analysis marked by vertical lines and labeled by subscripts), the constituent order of the second sentence is parallel to that of the first (i.e. subject-object) while in (4), the constituent order is non-parallel (i.e., object-subject).

(3) Vor einer Stunde bezwang | der Fechter (subj) $\left.\right|_{\text {NP1 }}$ den Gegner (obj) $\left.\right|_{\text {NP2 }}$ und gerade besiegt $\mid$ der Ringer (subj) $\left.\right|_{\text {NP3 }}$ den Erzfeind (obj) $\left.\right|_{\text {NP4 }}$, wie der Journalist schreibt. 'An hour ago defeated | the fencer (subj) $\left.\right|_{\mathrm{NP} 1}$ the adversary (obj) $\left.\right|_{\mathrm{NP} 2}$ and currently overwhelms $\mid$ the wrestler (subj) $\left.\right|_{\mathrm{NP} 3}$ the arch-enemy (obj) $\left.\right|_{\mathrm{NP} 4}$, as the journalist writes.'

(4) Vor einer Stunde bezwang $\mid$ den Fechter (obj) $\left.\right|_{\text {NP1 }}$ der Gegner (subj) $\left.\right|_{\text {NP2 }}$ und gerade besiegt $\mid$ der Ringer (subj) $\left.\right|_{\text {NP3 }}$ den Erzfeind (obj) $\left.\right|_{\text {NP4 }}$, wie der Journalist schreibt. 'An hour ago defeated $\mid$ the fencer (obj) $\left.\right|_{\mathrm{NP} 1}$ the adversary (subj) $\left.\right|_{\mathrm{NP} 2}$ and currently overwhelms $\mid$ the wrestler (subj) $\left.\right|_{\mathrm{NP} 3}$ the arch-enemy (obj) $\left.\right|_{\mathrm{NP} 4}$, as the journalist writes.'

(Knoeferle and Crocker 2009)

This study replicated previous findings of parallelism effects in English by Frazier and colleagues. Faster total (but not first-pass) reading times were observed for the regions containing the second conjunct (marked as NP3 and NP4 in (3-4) above) when the constituent order was parallel to that of the first conjunct. Furthermore, Knoeferle and Crocker found similar parallelism effects whether the syntactic structure was ambiguous or unambiguous (Exp. 1, 2; also see Frazier et al. 1984) and whether the constituent order was marked or unmarked (i.e., canonical) (Exp. 1, 2; but c.f. Frazier et al. 1984).

Based on the preceding experiments, parallelism effects can clearly be observed for a number of aspects of linguistic structure and across languages. The critical question, then, is what is the underlying source of these effects? One early hypothesis was that non-parallel coordinate structures are simply unacceptable because they violate a grammatical constraint on coordination (i.e., some version of Chomsky's original "like category constraint" (Chomsky 1957)); however, this theory was contradicted by an experiment that demonstrated parallelism effects in comparisons where the parallel and non-parallel constructions were both rated as fully acceptable (Frazier et al. 2000, Exp. 1). In addition, several experiments have shown evidence of parallelism effects related to structural features not proposed to be subject to grammatical constraints on coordination. For example, parallelism effects have been observed that were related to the internal structure of two conjoined noun phrases (Frazier et al. 2000, Exp. 3) and to aspects of semantic structure (e.g., animacy (Frazier et al. 1984).

Along these lines, a critical experiment by Knoeferle and Crocker (Knoeferle and Crocker 2009, Exp. 3), manipulated parallelism in not only syntactic structure (i.e., constituent order as described above) but also semantic structure (i.e., the subtle semantic similarity of constituents). For example, in sentence (3-4) above, "the fencer" is more similar to "the wrestler" (both terms describing a participant in a specific sport) than to "the arch-enemy" (a term describing a contestant more broadly). In this experiment, both syntactic and semantic parallelism were associated with parallelism effects, although the effect of syntactic parallelism was larger; moreover, the strongest parallelism effects occurred when both types of information were parallel, suggesting an additive nature to parallelism.

Taken together, the results described above suggest that parallelism effects arise from the processor rather than the grammar. The essential logic is that it is easier for the processor to process material that is structurally similar in some way to material it has recently processed. Although the exact nature of the processes that underlie parallelism effects has not yet been fully described, the pattern of results in previous studies is sufficient to reject two 
processing-based hypotheses that have been previously discussed. The first hypothesis is that parallelism effects result from an ambiguity resolution process. Under such a mechanism, when syntactic structure is temporarily ambiguous, the processor simply re-uses previously built structure, resulting in facilitated processing. This hypothesis makes one straightforward prediction that is disconfirmed by the literature: parallelism effects should only be observed in syntactically-ambiguous environments. In fact, several studies have observed parallelism effects in completely unambiguous constructions (Apel et al. 2007; Frazier et al. 1984, 2000; Knoeferle and Crocker 2009), so it must be concluded that parallelism effects do not result from the process of ambiguity resolution.

The second hypothesis is that parallelism effects result from the facilitation of early syntax-based parsing routines. This is a clear instantiation of the idea of facilitation based on previous processing and thus is an attractive hypothesis. Unfortunately, evidence of parallelism effects related to features that are not considered in an early structural analysis (e.g., animacy, discourse linking, semantic similarity) is problematic for this hypothesis. In addition, although effects related to parsing are often observable in the earliest measures of processing, parallelism effects have thus far only been observed in later measures (c.f. Frazier et al. 2000; Knoeferle and Crocker 2009). These facts point away from a facilitation of syntax-based parsing alone, although they do not completely preclude the possibility. The most convincing evidence against this hypothesis comes from the only restrictions on the scope of parallelism effects observed thus far. The only two previous experiments that failed to find parallelism effects were those that investigated parallelism in non-conjoined contexts (i.e., between subjects and objects, see Frazier et al. 2000, Exp. 4; Apel et al. 2007, Exp. 2), suggesting that parallelism effects are restricted to coordinate syntactic structures. Along these lines, a very interesting study (Knoeferle 2007) investigated whether parallelism effects in coordinate structures are tied to the use of specific conjunctions. They found parallelism effects in and-and while- conjoined contexts where the conjunction typically is associated with parallelism between the conjuncts, but not in but-conjoined contexts where it typically is associated with contrast between the conjuncts. This finding suggests a critical role for semantic or discourse information and a link between parallelism effects and the discourse relationship between the conjuncts as represented by a particular conjunction (c.f. Kehler 2002; Knoeferle and Crocker 2009; Shapiro and Hestvik 1995).

Since parallelism effects are restricted to some extent in terms of scope (i.e., to conjoined contexts where the conjunctions suggest parallelism of the conjuncts), parallelism effects cannot simply reflect the increased activation of recently processed structural material (i.e., the pure syntactic priming described above); nonetheless, it is still possible that parallelism effects are related to the activation of the previously processed parallel phrase. If the activated representation of the previously processed material contains syntactic and semantic information, then this suggestion would be consistent with findings that similarity in semantic structure can elicit parallelism effects.

\section{The Reactivation Hypothesis}

We suggest one intriguing theory about the processes involved in such a priming mechanism: the sustained reactivation hypothesis. This hypothesis makes use of a well-known context-driven memory-based reactivation process. This process has been demonstrated to be operative in sentence processing in the context of relations between phrases that refer to the same real-world entity or concept (i.e., an antecedent and an anaphor). Specifically, as suggested by numerous studies (see Nicol and Swinney 2002, for a review), the presentation of an anaphor elicits the immediate reactivation of the previously processed 
antecedent information from memory for integration with the surrounding context (a process reflected in priming for a probe related to the antecedent). Since the sustained reactivation hypothesis relies on a process already documented to be operative in sentence processing, it is attractively parsimonious. Furthermore, there is good reason to believe that similar processes might be involved in processing parallelism and anaphoric relationships. Antecedents and anaphors commonly occur as parallel elements, although they are not restricted to parallel environments, and parallelism has been proposed as an important mechanism in anaphoric interpretation (c.f. Chambers and Smyth 1998; Kehler 2002; Smyth 1994).

Essentially, in the context of parallelism, the sustained reactivation hypothesis proposes that, when a cue associated with parallelism is encountered in the context of coordinate structures, previously processed material is reactivated. This material then remains active until it can be integrated with material from the second conjunct. One consequence of this activation is facilitated processing for linguistic material that is parallel to the reactivated material on any of a variety of relevant dimensions. Specifically, the sustained reactivation hypothesis claims that, in coordinate structures, a conjunction that is associated with parallelism (e.g., and, see Knoeferle 2007, for details) elicits the reactivation of the previous conjunct so that the two conjuncts can be integrated; however, the sustained reactivation hypothesis is theoretically compatible with the idea that other linguistic (or potentially even non-linguistic) cues that are associated with parallelism could also elicit reactivation.

The current study investigates the sustained reactivation hypothesis by examining the activation of a parallel element in and-coordinated sentences such as (5) below.

(5) The doctor read the chart of the child with the broken arm during his morning rounds, and the insurance agent in the tacky suit did as well in order to become more familiar with the case.

In keeping with the majority of previous research on parallelism, this study tests contexts that involve parallelism between two verb phrases (VPs). The VP in the second conjunct is represented by the VP-anaphoric phrase, did as well, because using a VP-anaphoric phrase rather than a repeated VP is more natural in these contexts. Using cross-modal lexical priming techniques that are sensitive to the activation of words within a sentence, we evaluate the activation of the verb from the first conjunct (e.g., read) at various points throughout the sentence. The sustained reactivation hypothesis predicts that the verb will be reactivated in the second conjunct following the conjunction. Furthermore, this hypothesis predicts that the activation of the verb will be sustained throughout the second conjunct.

Verbs are a good candidate for testing sentence-level parallelism effects because they play a crucial syntactic and semantic role in the sentence. In addition to denoting the event, the verb carries information about the participants in the event and the roles they play. Furthermore, during sentence processing, verbs have been demonstrated to immediately activate not only their meaning (e.g., de Goede 2006; Callahan et al. 2009) but also typically associated arguments, argument structures, and thematic role information (Altmann and Kamide 1999; Boland et al. 1990; Ferretti et al. 2001; Friederici and Frisch 2000; McRae et al. 1997, 1998; Shapiro et al. 1987, 1989). Of even greater relevance to the current study, comprehenders have been demonstrated to use the information provided by verbs to facilitate processing by generating expectations about upcoming context (e.g., Altmann and Kamide 1999; Shapiro et al. 1993; Trueswell and Kim 1998; Trueswell et al. 1993). 


\title{
Experiment 1: Verb Activation Before and After the Conjunction
}

\author{
Methods
}

\section{Participants}

The participants in this and the following experiment were recruited from the University of California, San Diego and received course credit for their participation. All participants in both experiments were right-handed, neurologically healthy, and had normal hearing and normal or corrected-to-normal vision. All participants were also native English speakers with no exposure to a second language before age six. Thirty-nine participants were initially tested in Experiment 1; however, five participants were excluded from further analysis for error rates on the on-line naming task that exceeded $10 \%$ on experimental items or $15 \%$ on all items. In addition, data from one participant were lost due to computer error. Therefore, 33 participants were included in further analysis for Experiment 1 ( 4 male, 25 female, 4 unknown ${ }^{2}$ mean age $=20.4$, range $=18-34$ ).

\section{Materials}

For the critical experimental items, 40 items of similar form were constructed. (Please see the Appendix for a full list). Each experimental item consisted of two sentences joined by the conjunction, and. The first conjunct began with a subject followed by a VP. The second conjunct began with a different subject followed by an anaphoric phrase (e.g., did too, did the same, did as well, etc.). Anaphoric phrases were used in this study because they occur very frequently in parallel constructions and typically sound more natural than a repetition of the VP from the first conjunct (a variety was used to lessen expectation effects). In addition, using anaphoric phrases made it possible to test downstream points because the verb was not directly presented in the second conjunct. A sample sentence appears in (6) below with the critical verb in italics.

(6) The doctor read the chart of the child with the broken $\operatorname{arm}^{1}$ during his morning rounds, and ${ }^{2}$ the insurance agent in the tacky suit did as well in order to become more familiar with the case. (Probes: reviewed (R), reserved (C))

For each experimental item, related (R) and control (C) probe words were selected (see (6) above for examples). Since the activation of a verb was being tested, all probe words were verbs. Related probe words (e.g., reviewed) were semantically related to the verb (e.g., read) in the first conjunct and were presented in the same tense (except one probe, swallow, which due to an oversight was presented in present rather than past tense). Control probe words (e.g., reserved) were also presented in the same tense and were matched to related probe words on length, frequency, and mean naming reaction time in an unprimed context, but critically were not semantically related to the critical verb or any other word preceding the test points. A pre-test was conducted to confirm that related probe verbs were strongly associated with the critical verb and control probe verbs were not. A separate group of 48 participants who did not participate in Experiments 1 or 2 were presented with 80 pairs of verbs. Each critical verb (e.g., read) was presented twice, once paired with the related probe verb (e.g., reviewed) and once with the control probe verb (e.g., reserved). Participants rated

2 Unfortunately, due to an error, gender and age information as well as comprehension scores for four participants in this experiment were lost. 
how associated each pair of verbs was using a Likert scale ranging from 1 (not associated at all) to 5 (very associated). Related probe verbs were rated as being highly associated with the critical verbs $($ Mean $=4.10 ; \mathrm{SD}=.36)$ while control probe verbs were not $(\mathrm{Mean}=1.48 ; \mathrm{SD}$ $=.25)$. Paired two-tailed t-tests revealed that this result was highly significant by participants and items $\left(t_{1}(47)=53.44, p=.000 ; t_{2}(39)=25.16, p=.000\right) .^{3}$

During the uninterrupted aural presentation of the experimental items, probe words were presented visually at one of two points (signified by superscripts ${ }^{1}$ and $^{2}$ above), the positions of which varied based on the experiment. In Experiment 1, the two probe points occurred in the first conjunct: (a) approximately halfway between the offset of the verb in the first conjunct and the onset of the conjunction (PP1) and (b) immediately following the conjunction (PP2). To minimize the possibility that participants would develop strategies during testing, 40 filler items were also constructed that consisted of two conjoined sentences. For filler items, probe type and probe position did not vary; the same probe word was always presented at the same probe point. This probe point occurred randomly throughout the filler sentences so that participants did not develop expectations about when the probe would be presented.

Using digital recording and editing software (Cool Edit Pro), all sentences were recorded by a female native English speaker at a normal volume and speaking rate. Probe positions were created by playing a $1 \mathrm{kHz}$ square-wave-onset tone on a parallel channel to that containing the speech (a channel not audible to participants). This tone was used by the computer program (RTLAB v.1.1) to initiate presentation of a visual word probe (for the naming task) and to begin response timing (which was terminated by the participant's naming response). Timing for the placement of the probe points was chosen by using Cool Edit Pro software to identify the proper point in the sentence.

The 40 experimental and 40 filler items were assembled into a single pseudo-randomized list with the constraint that no experimental condition ever appeared twice in a row. Four counterbalanced versions of the list were created by systematically varying probe type and probe position across the lists for the experimental items. Although each item only appeared once in a given list, across lists each item was paired with a probe from each condition. Participants responded to probes in all four conditions, but only heard any given item once. Thus, over the course of the experiment, each participant was presented with 40 items from the four critical conditions as well as 40 filler items. In addition, five multiple-choice comprehension questions were randomly distributed throughout the list to make certain that participants were attending to the sentences (e.g., Was the sentence you just heard about...? (a) a corporal (b) an admiral (c) a sergeant). An experimenter read the questions and answer choices at the proper points and participants responded by circling the appropriate letter on an answer sheet. Questions were always followed by a filler item in case responding to the question resulted in a disruption of processing.

\section{Procedure}

Before beginning the experiment, participants gave informed consent and completed a questionnaire about their language use and language experience. Next, the participants received instructions for the cross-modal naming task. Participants were informed that they would be listening to sentences and that at some point during the sentence a word would appear on the screen. Participants were instructed to then say the word out loud as quickly as possible.

3 The control probes for three items in this pre-test were later changed to better match the a priori naming time of the related probe. Removing these items did not change these effects. 
Participants were also told that after some of the sentences the experimenter would ask them a comprehension question and that they should circle the correct answer on their answer sheet. Once participants indicated that they understood the instructions, the experiment began.

Participants sat facing a PC-compatible computer running RTLAB v.1.1 that controlled the presentation and data collection with millisecond accuracy. Each participant was exposed to one version of the four counterbalanced lists containing 40 experimental items and 40 filler items. The first ten sentences of each session were always filler sentences and served as practice trials to familiarize the subjects with the procedure. Each item was presented aurally through headphones and the appropriate visual probe was displayed on the computer screen at the appropriate point while participants' voiced responses were recorded via microphone by the computer and a SONY digital tape recorder. Timing was initiated along with the onset of the visual probe and stopped by the onset of the response. The entire experiment lasted less than $1 \mathrm{~h}$.

\section{Experimental Design}

In this $2 \times 2$ multi-factorial design, the critical conditions were considered as part of a repeated-measures design with two within-subjects factors, Probe Point (PP1 or PP2) and Probe Type (Related or Control).

Results

Participants were highly accurate in their responses to comprehension questions (Mean: 94.48\%; SD: 9.1\%). The probe naming response times were initially screened to remove probes for which no response was recorded or for which the experimenter determined the response to be inappropriate. This initial screen resulted in the removal of 28 data points (or less than $2 \%$ of the data), roughly equally distributed across conditions. Subsequent to this screening, a repeated-measures ANOVA was conducted involving the factors Probe Point (PP1 or PP2) and Probe Type (Related or Control). Please see Table 1 for means and standard deviations.

In Table 1, the difference was calculated by subtracting the mean response time for Related probes at a given probe point from the mean response time for Control probes at the same probe point; however, in the table, the difference does not always equal the subtraction of the control and related because of rounding. A positive difference indicates faster response times

Table 1 Means and standard deviations in terms of response time (ms) for Experiments 1 and 2

\begin{tabular}{|c|c|c|c|c|}
\hline \multirow[t]{2}{*}{ Probe type } & \multicolumn{4}{|l|}{ Probe point } \\
\hline & $1(n=31)$ & $2(n=31)$ & $3(n=33)$ & $4(n=33)$ \\
\hline \multirow[t]{2}{*}{ Control } & 632 & 619 & 647 & 624 \\
\hline & (74) & (78) & (126) & (117) \\
\hline \multirow[t]{2}{*}{ Related } & 633 & 590 & 614 & 594 \\
\hline & (107) & (83) & (103) & (110) \\
\hline Difference & $-1^{\mathrm{ns}}$ & $29^{*}$ & $32 *$ & $31^{*}$ \\
\hline
\end{tabular}

Note: Paired $t$-tests revealed that differences marked with a star $(*)$ were significant at $p<.05$ while those marked "ns" were not $(p>.05)$. Probe points 1 and 2 were tested in Exp. 1 while Probe points 3 and 4 were tested in Exp. 2 
for Related than Control probes (i.e., priming) and a negative difference indicates slower response times for Related than Control probes. A comparison of the response times for Related and Control probes suggested priming at PP2 (29 ms advantage for related probes), but not at PP1 (1 ms advantage for control probes). The statistical analysis generally confirmed this pattern by revealing a main effect of Probe Point $\left(F_{1}(1,32)=15.41, p=\right.$ $\left..000, F_{2}(1,39)=6.78, p=.01\right)$, but no main effect of Probe Type $\left(F_{1}(1,32)=2.82, p=\right.$ $\left..10, F_{2}(1,39)=3.30, p=.08\right)$. In addition, the interaction between Probe Point and Probe Type was numerically supported and reached significance in the subject, but not the item analysis $\left(F_{1}(1,32)=5.02, p=.03, F_{2}(1,39)=1.10, p=.30\right)$. Based on a-priori hypotheses, paired one-tailed t-tests were also conducted comparing Related and Control probes at each probe point. These analyses further confirmed a significant difference between the two probe types at PP2 $\left(t_{1}(32)=4.44, p=.000, t_{2}(39)=1.92, p=.03\right)$, but not at PP1 $\left(t_{1}(32)=.10, p=.45, t_{2}(39)=.47, p=.32\right)$.

\section{Discussion}

In Experiment 1, significant priming for the verb was observed at PP2, but not at PP1. The failure to find priming at PP1 suggests that the activation of the verb related to its initial presentation decayed by, on average, approximately $1,600 \mathrm{~ms}$ later. This observation fits with previous priming experiments that suggest that the initial activation of a word decays relatively quickly after presentation (e.g., Love and Swinney 1996). Of greater relevance, the pattern of results supports the sustained reactivation hypothesis since reactivation was temporally linked to the presentation of the conjunction (i.e., and). Our second experiment was designed to further evaluate this hypothesis by continuing to chart the activation of the verb in the second conjunct. The findings of both experiments will be interpreted in the General Discussion to follow Experiment 2.

\section{Experiment 2: Verb Activation Throughout the Second Conjunct}

Having confirmed that reactivation occurs, we now evaluate its time-course or duration. The sustained reactivation hypothesis claims that reactivated information is sustained until it can be completely integrated (i.e., at least until the end of the second conjunct). Experiment 2 investigates this hypothesis by further inspecting the pattern of priming in the second conjunct. Specifically, two later points in the second conjunct were tested: (a) $700 \mathrm{~ms}$ before the onset of the parallel element in the second conjunct (i.e., the VP-anaphoric phrase) (PP3) and (b) immediately following this phrase (i.e., at the end of the second conjunct) (PP4). Thus, PP3 and PP4 occurred on average approximately 1,100 and 2,500 ms after PP2. Failing to find priming at either PP3 or PP4 would constitute evidence against the sustained reactivation hypothesis, while finding priming at both PP3 and PP4 would constitute evidence for this hypothesis.

\section{Methods}

\section{Participants}

Thirty-nine participants were initially tested in Experiment 2; however, five participants were excluded from further analysis for error rates on the on-line naming task that exceeded $10 \%$ on experimental items or $15 \%$ on all items. In addition, data from three participants was 
lost due to computer error. Therefore, 31 participants were included in further analysis for Experiment 2 (14 male, 17 female; mean age $=19.6$, range $=18-25$, with one participant failing to provide age information).

\section{Materials and Procedure}

With the exception of a slight change in the wording of two items to improve clarity, the materials, task, and procedure were identical to those used in Experiment 1 and have been described above. The only modification in Experiment 2 was the placement of the probe points. In Experiment 2, the two probe points were chosen to investigate whether the priming of the verb is maintained throughout the second conjunct and occurred (a) $700 \mathrm{~ms}$ before the onset of the parallel element in the second conjunct (i.e., the VP-anaphoric phrase) (PP3) and (b) immediately following this phrase (i.e., at the end of the second conjunct (PP4). Thus, PP3 and PP4 occurred on average approximately 1,100 and 2,500 ms after PP2. These points are marked with superscripts 3 and 4 in the sample sentence in (7) below.

(7) The doctor read the chart of the child with the broken $\operatorname{arm}^{1}$ during his morning rounds, and ${ }^{2}$ the insurance agent in $^{3}$ the tacky suit did as well ${ }^{4}$ in order to become more familiar with the case. (Probes: reviewed (R), reserved (C))

\section{Experimental Design}

In this $2 \times 2$ multi-factorial design, the critical conditions were considered as part of a repeated-measures design with two within-subjects factors, Probe Point (PP3 or PP4) and Probe Type (Related or Control).

Results

Participants were highly accurate in their responses to comprehension questions (Mean: 93.55\%; SD: $10.82 \%$ ). The probe naming response times were initially screened to remove probes for which no response was recorded or for which the experimenter determined the response to be inappropriate. This initial screen resulted in the removal of 17 responses (or approximately $1.4 \%$ of the data), roughly equally distributed across conditions. Subsequent to this screening, a repeated-measures ANOVA was conducted involving the factors Probe Point (PP3 or PP4) and Probe Type (Related or Control). Please see Table 1 for means and standard deviations.

A comparison of the response times for Related and Control probes suggested priming at PP3 (32 ms advantage for related probes) and PP4 (31 ms advantage for related probes). The statistical analysis generally confirmed this pattern by revealing a main effect of Probe Point $\left(F_{1}(1,30)=13.28, p=.001, F_{2}(1,39)=6.03, p=.02\right)$ and a main effect of Probe Type $\left(F_{1}(1,30)=11.47, p=.002, F_{2}(1,39)=9.32, p=.004\right)$. The interaction between Probe Point and Probe Type did not reach significance $\left(F_{1}(1,30)=.02, p=.89, F_{2}(1,39)=\right.$ $.19, p=.67)$. Based on a-priori hypotheses, paired one-tailed t-tests were also conducted comparing Related and Control probes at each probe point. These analyses further confirmed a significant difference between the two probe types at PP3 $\left(t_{1}(30)=2.34, p=\right.$ $\left..01, t_{2}(39)=2.94, p=.003\right)$ and PP4 $\left(t_{1}(30)=3.82, p=.001, t_{2}(39)=2.02, p=.03\right)$. 
Discussion

In Experiment 2, consistent with the sustained reactivation hypothesis, priming effects were observed at both PP3 and PP4. Considering the results from both experiments, priming for the verb from the first conjunct was observed at all points tested in the second conjunct (i.e., PP2, PP3, and PP4), indicating that the activation of the verb was sustained at least approximately $2,500 \mathrm{~ms}$ after its initial reactivation following the conjunction. In addition, the numerical magnitude of the priming effect was virtually identical across the three probe points where it was detected (PP2, PP3, and PP4). The implication of these findings will be discussed in the General Discussion.

\section{General Discussion}

Previous studies have demonstrated that the processing of the second conjunct of a conjoined phrase is facilitated if it is parallel to the first conjunct (Apel et al. 2007; Frazier et al. 1984, 2000; Knoeferle 2007; Knoeferle and Crocker 2009), The current study investigated a specific hypothesis about the processes underlying these parallelism effects, the sustained reactivation hypothesis, by examining the activation of a parallel element (i.e., a verb) throughout two "and"-coordinated sentences. We examined evidence of activation (i.e., priming as indicated by faster response times for semantically related vs. unrelated words) at four points throughout the sentences: (1) approximately 1,600 $\mathrm{ms}$ after the presentation of the verb in the first conjunct (PP1), (2) immediately following the conjunction (PP2), (3) approximately $1,100 \mathrm{~ms}$ after the conjunction (PP3), (4) at the end of the second conjunct (i.e., approximately $2,500 \mathrm{~ms}$ after the conjunction) (PP4).

In the context of previous research, the results at PP1 reveal the time-course of the activation of the verb in the first conjunct. It is well-known that processing a word immediately activates its lexical representation. For a verb, this representation includes meaning information (de Goede 2006; Callahan et al. 2009) as well as associated semantic and syntactic information (Altmann and Kamide 1999; Boland et al. 1990; Ferretti et al. 2001; Friederici and Frisch 2000; McRae et al. 1997, 1998; Shapiro et al. 1987, 1989). Based on previous research suggesting that activation related to the presentation of a word decays relatively quickly (e.g., Love and Swinney 1996; Nicol and Swinney 1989), we predicted that verb activation would not be detected at PP1. Confirming this prediction, no priming was observed at PP1, suggesting that the activation of the verb related to the initial presentation decayed (at least by approximately 1,600 ms later). Despite being consistent with previous research on noun and verb processing in English sentences, it is worth noting this finding is inconsistent with a study that investigated verb processing in Dutch sentences and found sustained activation throughout the entire clause related to the verb (de Goede et al. 2009); however, the Dutch study differed from previous studies and the current study in a number of ways. In particular, we suspect that the fact that in the Dutch experimental sentences the verb was displaced from the canonical position via head movement might be relevant to the observed pattern of activation. Since head movement is a relatively unexplored psycholinguistic phenomenon, further research is needed to shed light on this issue.

In the context of previous research, the results at PP2, PP3, and PP4 further clarify the activation of the verb in the second conjunct. The sustained reactivation hypothesis claims that encountering a cue associated with parallelism (e.g., the conjunction, and) elicits the reactivation of previously processed relevant material. Based on this hypothesis, we predicted a priming effect at PP2 immediately following the conjunction. Experiment 1 confirmed this pre- 
diction, revealing a significant $29 \mathrm{~ms}$ advantage for related probes at PP2. Experiment 2 then investigated the activation of the verb at later points in the second conjunct. The sustained reactivation hypothesis predicted that the reactivation of the verb would be maintained throughout the second conjunct (i.e., priming effects at PP3 and PP4). Indeed, the results of Experiment 2 revealed a significant 32 and $31 \mathrm{~ms}$ advantage for related probes at PP3 and PP4 respectively.

It is worth noting that the numerical magnitude of the priming effect in the current study was similar across the three points tested in the second conjunct. Residual activation due to incomplete decay would be associated with a reduction in the magnitude of the priming effect (Shapiro and Hestvik 1995). Thus, the nearly identical priming effects at PP2, PP3, and PP4 suggest sustained activation due to active maintenance rather than residual activation due to incomplete decay. Active maintenance processes that are linked to short-term memory, as reflected electrophysiologically by a sustained left anterior negativity (c.f. King and Kutas 1995), have been observed previously in sentence processing when antecedent information cannot be immediately integrated (e.g., as in relations involving displaced arguments). Since the cue to parallelism preceded the processing of the second conjunct in the current study, information from the first conjunct could not be integrated immediately following reactivation.

The results of Experiments 1 and 2 suggest the following conclusions about the activation pattern of a verb in "and"-coordinated sentences: (1) decay occurs relatively quickly following initial activation, (2) the conjunction elicits reactivation, and (3) this reactivation is sustained until at least the end of the second conjunct. As discussed previously, a successful psycholinguistic theory of parallelism must describe not only the nature and time-course of the underlying processes but also the relevant mechanisms and underlying representations. The sustained reactivation hypothesis suggests that two processes independently demonstrated to be involved in sentence processing (i.e., reactivation and maintenance) play a key role in generating parallelism effects. Specifically, this hypothesis claims that, in coordinate structures, a cue that is associated with parallelism (e.g., and, see Knoeferle 2007) elicits the reactivation of previously processed material from the first conjunct so that this information can be integrated with information from the second conjunct. Following reactivation, the activation of the material is maintained at least until integration can be completed (i.e., until the end of the second conjunct). Although the sustained reactivation hypothesis points to the conjunction, and, as the relevant cue to parallelism in the current study (and in many previous studies), the notion of cue is intended to be broad enough to encompass other linguistic (or potentially even non-linguistic) cues to parallelism.

Having found support for a hypothesis regarding the nature and time-course of the processes underlying parallelism effects, we now delineate some important remaining questions about a theory of parallelism and suggest some tentative extensions of the current hypothesis. One remaining question concerns the mechanism that underlies the reactivation process. A series of studies by McElree and colleagues (McElree 2000; McElree et al. 2003; Martin and McElree 2008) have suggested that reactivation in several types of linguistic dependencies (e.g., long-distance anaphoric relationships, subject-verb relationships) is accomplished via a cue-driven, direct access mechanism: we suggest that such a mechanism may also underlie the reactivation process in parallelism with the conjunction serving as the relevant cue. As Martin and McElree (2008) note, this mechanism is generally consistent with copy $\alpha$, a structure sharing mechanism proposed by Frazier and Clifton (2001) to underlie the processing of coordinate structures involving verb-phrase ellipsis; however the cue-driven direct access mechanism has the advantage of not being restricted to certain types of constructions. Another question relates to the types of information that are reactivated following the conjunction (e.g., phonological, syntactic, semantic, discourse, etc.). Since both syntactic and 
semantic parallelism effects have been observed, the reactivated representation must contain at the very least both of these types of information; however, since parallelism can occur at a number of levels of structure ranging from phonology to discourse, we go a step further and suggest that the reactivated representation may contain information at each of these levels.

One final question is whether all parts of a complex first conjunct are reactivated. Based on the results of the current study, we can confidently state that the verb is activated in coordinated sentences; however, as stated previously, we chose to test the activation of the verb because it plays a particularly important role in the sentence. The question remains, then, whether less critical parts of the conjunct are reactivated. Interestingly, there have been several priming experiments involving similar parallel constructions (Shapiro and Hestvik 1995; Shapiro et al. 2003) that have investigated the activation of the subject or object from the first conjunct. The authors interpreted the results as suggesting that the subject and object of the VP in the first conjunct were not activated at a point downstream from the conjunction in the second conjunct but were reactivated following a VP-anaphoric phrase at the end of the second conjunct. Despite their interpretation, we note that the effect for the object at the earlier point did reach the standard level of significance at $p=.05$ ( $p=.01$ was the required level when corrected for multiple comparisons), so it is possible that the object was indeed activated at this point. Regardless, although these data do pose a problem for a strong version of the sustained reactivation hypothesis that claims that all potentially parallel material is reactivated following the conjunction and maintained until it can be fully integrated, they are compatible with a more incremental version of this hypothesis whereby individual constituents decay following integration with their parallel counterparts or a version where only certain parts of the first conjunct are reactivated.

Although much progress has been made towards a theory of parallelism, future research will be critical in refining and solidifying the tentative conclusions described above. In particular, investigations of parallelism that evaluate the role of specific conjunctions as well as other linguistic cues will allow the scope of parallelism to be defined more clearly. Similarly, investigations of the activation patterns of different components of complex coordinate structures (e.g., the subject, the object, adjunct phrases, etc.) and different types of information (e.g., phonological, syntactic, semantic, discourse, etc.) will allow more confident statements regarding the characteristics of the reactivated representation.

Acknowledgments During the completion of this study, the first author was supported by an NSF Graduate Research Fellowship as well as a traineeship awarded by NIH to the Center for Research in Language at the University of California, San Diego. This research was also funded by NIH-DC000494.

Open Access This article is distributed under the terms of the Creative Commons Attribution Noncommercial License which permits any noncommercial use, distribution, and reproduction in any medium, provided the original author(s) and source are credited.

\section{Appendix}

1. The admiral lectured on submarines last Saturday in a speech to his men, and the President of the United States did the same a week later in Congress.

$$
\text { R: spoke C: added }
$$

2. Parisians drink wine which is native to the country, and farmers throughout the Provinces do too because of the high quality and cheap prices available.

$$
\text { R: swallow C: skipped }
$$


3. The artist improved the design until it was ready to sell, and the businessman in the nearby town did the same with the new line of clothes.
$\mathrm{R}$ : perfected
C: suspected

4. The secretary chatted on the telephone at the desk in the front office all day long, and the Vice President in the adjoining office did too because his assistant was on vacation.
R: talked
C: topped

5. Athletes train muscle pattern responses necessary for maximum performance, and musicians in symphonic ensembles do the same while rehearsing the more difficult passages of each piece.
$\mathrm{R}$ : practiced
C: described

6. The journalist wrote on Sunday despite the fact that it was here day off, and the researcher in the Neurosciences Department did as well for an article being presented at a conference.

$$
\text { R: typed C: named }
$$

7. The equestrian trained the quarter horse that was a strong contestant in the race, and the cowboy from a Texas ranch did as well in order to keep the horse in good shape.

$$
\text { R: taught } \quad \mathrm{C} \text { : joined }
$$

8. The girl looked for a gift for the birthday party, and the receptionist at the large firm did the same because the boss was always so busy.

$$
\mathrm{R} \text { : searched } \mathrm{C} \text { : reserved }
$$

9. The mime performed for the tourists on the pier, and the punk rocker with the rainbow hair did too every Sunday afternoon.

$$
\mathrm{R}: \text { acted } \mathrm{C} \text { : raced }
$$

10. The hunter shot a rattlesnake in the lot around the corner, and the animal control officer in the neighborhood did too providing a safe environment for after school play.

$$
\text { R: killed C: walked }
$$

11. The doctor read the chart of the child with the broken arm during his morning rounds, and the insurance agent in the tacky suit did as well in order to become more familiar with the case.
$\mathrm{R}$ : reviewed
C: reserved

12. The old man observed the children in the park on sunny afternoons, and the office workers from the corporate annex did the same during their lunch breaks.
$\mathrm{R}$ : watched
C: figured

13. The priest skipped breakfast Tuesday morning because of the time change, and the electrician from up the street did as well because he had to run errands in the morning.
R: missed
C: topped 
14. The farmer fed the animals before going to bed, and the veterinarian who runs the emergency clinic did too before leaving work for the evening.
R: starved
C: groaned

15. The jeweler examined the rock to see if it was a precious stone, and the geologist who was on a trip to Africa did the same while trying to identify its origin.
R: studied
C: decided

16. The cyclist crossed the street in the middle of rush hour traffic, and the cabby from New York City did the same on his way to the airport.
$\mathrm{R}$ : traversed
C: elongated

17. The shaman explored the desert terrain in search of particular roots, and the hikers from the East Coast did as well on their vacation to Joshua Tree.

$$
\mathrm{R} \text { : pioneered } \mathrm{C} \text { : compacted }
$$

18. The chemist discovered the ingredients that were necessary for his experiment, and the witch doctor from a Haitian village did the same because he was intent upon casting his spell.

$$
\text { R: found } \quad \mathrm{C} \text { : faced }
$$

19. The Queen of England toured the countryside in order to promote good will among the farmers, and the professor from the archaeology department did as well in order to learn about the area.

$$
\text { R: traveled C: suspended }
$$

20. The engineer entered the field as a troubleshooter for his company, and the social worker from Child Welfare Services did as well to assess the progress made in her cases.

$$
\text { R: exited C: dialed }
$$

21. The dictator put people in jail by the thousands in order to suppress insurrection, and the judge on the State judiciary board did too after 50 years on the bench.

$$
\text { R: confined C: deserted }
$$

22. The postman cruised the neighborhood to deliver the mail to each house, and the TV repairman from the cable company did too while looking for the correct addresses.

$$
\text { R: drove C: meant }
$$

23. The chef baked a cake for the annual competition, and the housewife in her brand new kitchen did the same for the birthday party.

$$
\text { R: prepared C: accepted }
$$

24. The critic hated the film shown last Saturday because of its flagrant inconsistencies, and the steelworker from Detroit did too because of the excessive violence involved.
$\mathrm{R}$ : loved
C: heard 
25. The glassblower created beautiful objects for the Ancient Artisans symposium, and the sculptor from Manhattan did as well, experimenting with modern and innovative techniques.

$$
\text { R: crafted C: gripped }
$$

26. The forest ranger looked at the compass to orient himself without sunlight, and the navigator of the Princess cruiseliner did the same to ensure he was not off course.

$$
\mathrm{R} \text { : viewed } \mathrm{C} \text { : shared }
$$

27. The miser ate the burger slowly to savor it as long as possible, and the school children at McDonald's did too while competing to see who chewed each bite the longest.

$$
\text { R: chewed C: matted }
$$

28. The paramedics looked at the scene of the accident to see if the victims were still alive, and the bystanders from across the street did the same because they were hoping for a good view.

$$
\text { R: scanned C: clipped }
$$

29. The librarian heard the announcement over the intercom system, and the jockey from the local stables did too when it was time for him to join the line-up for the upcoming race.

$$
\mathrm{R} \text { : listened } \quad \mathrm{C} \text { : intended }
$$

30. The plumber purchased the Big Gulp at 7-11 on his way home from work, and the comedian in the VW bug did too because she was thirsty for a cold drink.

$$
\text { R: bought } \quad \mathrm{C} \text { : jumped }
$$

31. The Catholic nun called the number to City Hall to schedule a room for the bible study, and the alcoholic from Kansas did the same so he could verify an appointment scheduled for next week

$$
\text { R: phoned C: coated }
$$

32. The botanist placed the plant in the sunlight after finding just the right spot, and the decorator from the Prospect Avenue firm did too because the location by the window was much nicer.

$$
\text { R: put C: let }
$$

33. The fireman took off the heavy boots that were covered in mud and ash, and the mountain climber from the French Alps did as well when he returned to camp.

$$
\mathrm{R} \text { : removed } \mathrm{C} \text { : charged }
$$

34. The ophthalmologist gave the jewelry to the museum in an attempt to keep it from a despised sibling, and the collector of Post-WW1 artifacts did the same knowing that many would appreciate their beauty.
R: offered
C: crowded 
35. The graduate student set the books on a shelf to deal with them later, and the accountant from the office did too so he would know where to begin in the morning.

$$
\text { R: placed C: ranted }
$$

36. The rock star promised the proceeds to charity when touring through LA last year, and the millionaire from the marketing firm did too when trying to build a better public image.

$$
\text { R: pledged C: spanned }
$$

37. The dogcatcher gave a puppy to the child in the dirty dungeon, and the grandfather from a ranch in Montana did the same during a visit to the family.

R: returned C: mattered

38. The principal addressed the problems of the students during the assembly period in the auditorium, and the police officer from the Public Relations Department did the same for a discussion about drug abuse.

$$
\mathrm{R} \text { : discussed } \mathrm{C} \text { : suggested }
$$

39. The housekeeper loaned the recipe to a neighbor for a dinner party the following evening, and the actress in need of publicity did too after making a big deal about how fantastic the food was.

$$
\text { R: borrowed C: glimpsed }
$$

40. The astronaut gave the clothing to the space museum for a re-opening next week, and the designer for the Globe Theater did the same with some of the older priceless costumes.

$$
\mathrm{R} \text { : donated } \mathrm{C} \text { : thrived }
$$

\section{References}

Altmann, G. T. M., \& Kamide, Y. (1999). Incremental interpretation at verbs: Restricting the domain of subsequent reference. Cognition, 73, 247-264.

Apel, J., Knoeferle, P., \& Crocker, M. W. (2007). Processing parallel structure: Evidence from eye tracking and a computational model. In S. Vosniadou, D. Kayser \& A. Protopapas (Eds.), In Proceedings of the 2nd European cognitive science conference (pp.125-131). London: Taylor \& Francis.

Boland, J. E., Tanenhaus, M. K., \& Garnsey, S. M. (1990). Evidence for the immediate use of verb control information in sentence processing. Journal of Memory and Language, 29, 413-432.

Callahan, S. M., Shapiro, L. P., de Goede, D., Wester, F., \& Love, T. (2009). An investigation of verb activation during sentence processing. Manuscript in preparation.

Carlson, K. (2001). The effects of parallelism and prosody in the processing of gapping structures. Language and Speech, 44, 1-26.

Chambers, C. G., \& Smyth, R. (1998). Structural parallelism and discourse coherence: A test of centering theory. Journal of Memory and Language, 39, 593-608.

Chomsky, N. (1957). Syntactic structures. The Hague: Mouton.

de Goede, D. (2006). Verbs in spoken sentence processing: Unraveling the activation pattern of the matrix verb. Groningen Dissertations in Linguistics, 63.

de Goede, D., Shapiro, L. P., Wester, F., Swinney, D. A., \& Bastiaanse, R. (2009). The time course of verb processing in Dutch sentences. Journal of Psycholinguistic Research, 38, 181-199.

Ferretti, T. R., McRae, K., \& Hatherell, A. (2001). Integrating verbs, situation schemas, and thematic role concepts. Journal of Memory and Language, 44, 516-547.

Frazier, L., \& Clifton, C. (2001). Processing coordinates and ellipsis: Copy $\alpha$. Syntax, 4(1), 1-22.

Frazier, L., Munn, A., \& Clifton, C. (2000). Processing coordinate structures. Journal of Psycholinguistic Research, 29, 343-370. 
Frazier, L., Taft, L., Roeper, T., Clifton, C., \& Ehrlich, K. (1984). Parallel structure: A source of facilitation in sentence comprehension. Memory \& Cognition, 12, 421-430.

Friederici, A. D., \& Frisch, S. (2000). Verb argument structure processing: The role of verb-specific and argument-specific information. Journal of Memory and Language, 43, 476-507.

Kehler, A. (2002). Coherence, reference, and the theory of grammar. Stanford, CA, USA: CSLI Publications.

King, J. W., \& Kutas, M. (1995). Who did what and when? Using word- and clause-level ERPs to monitor working memory usage in reading. Journal of Cognitive Neuroscience, 7, 376-395.

Knoeferle, P. (2007). Eye tracking parallelism effects in coordinate constructions: The effects of "and", "but" and "while". Poster presented at the 20th Annual CUNY. La Jolla, USA.

Knoeferle, P., \& Crocker, M. W. (2009). Constituent order and semantic parallelism in online comprehension: Eye-tracking evidence from German. Quarterly Journal of Experimental Psychology. doi:10.1080/ 17470210902790070.

Love, T. E., \& Swinney, D. A. (1996). Coreference processing and levels of analysis in object-relative constructions: Demonstration of antecedent reactivation with the cross-modal priming paradigm. Journal of Psycholinguistic Research, 25, 5-24.

Martin, A. E., \& McElree, B. (2008). A content-addressable pointer mechanism underlies the comprehension of verb-phrase ellipsis. Journal of Memory and Language, 58, 879-906.

McElree, B. (2000). Sentence comprehension is mediated by content-addressable memory structures. Journal of Psycholinguistic Research, 29, 111-123.

McElree, B., Foraker, S., \& Dyer, L. (2003). Memory structures that subserve sentence comprehension. Journal of Memory and Language, 48, 67-91.

McRae, K., Ferretti, T. R., \& Amyote, L. (1997). Thematic roles as verb-specific concepts. Language and Cognitive Processes, 12, 137-176.

McRae, K., Spivey-Knowlton, M. J., \& Tanenhaus, M. K. (1998). Modeling the influence of thematic fit (and other constraints) in on-line sentence comprehension. Journal of Memory and Language, 38, 283-312.

Nicol, J., \& Swinney, D. (1989). The role of structure in coreference assignment during comprehension. Journal of Psycholinguistic Research, 18, 5-19.

Nicol, J., \& Swinney, D. (2002). The psycholinguistics of anaphora. In A. Barss (Ed.), Anaphora (pp. $72-$ 104). Oxford: Blackwell Press.

Shapiro, L. P., \& Hestvik, A. (1995). On-line comprehension of VP-ellipsis: Syntactic reconstruction and semantic influence. Journal of Psycholinguistic Research, 24, 517-532.

Shapiro, L. P., Hestvik, A., Lesan, L. A., \& Garcia, A. R. (2003). Charting the time course of VP-ellipsis sentence comprehension: Evidence for an initial and independent structural analysis. Journal of Memory and Language, 49, 1-19.

Shapiro, L. P., Nagel, H. N., \& Levine, B. A. (1993). Preferences for a verb's complements and their use in sentence processing. Journal of Memory and Language, 32, 96-114.

Shapiro, L. P., Zurif, E., \& Grimshaw, J. (1987). Sentence processing and the mental representation of verbs. Cognition: International Journal of Cognitive Science, 27, 219-246.

Shapiro, L. P., Zurif, E. B., \& Grimshaw, J. (1989). Verb processing during sentence comprehension: Contextual impenetrability. Journal of Psycholinguistic Research, 18, 223-243.

Smyth, R. (1994). Grammatical determinants of ambiguous pronoun resolution. Journal of Psycholinguistic Research, 23, 197-229.

Trueswell, J. C., \& Kim, A. E. (1998). How to prune a garden path by nipping it in the bud: Fast priming of verb argument structure. Journal of Memory and Language, 39, 102-123.

Trueswell, J. C., Tanenhaus, M. K., \& Kello, C. (1993). Verb-specific constraints in sentence processing: Separating effects of lexical preference from garden-paths. Journal of Experimental Psychology: Learning, Memory, and Cognition, 19, 528-553. 